\title{
Long-term effects of food deprivation: I. Impact on pain reactivity and shock-induced hypoalgesia
}

\author{
PAUL A. ILLICH, ANDREA R. ALLEN, and JAMES W. GRAU \\ Texas A\&M University, College Station, Texas
}

\begin{abstract}
Prior work has shown that the uncontrollable shock procedure used to induce learned helplessness produces a hormonally mediated opioid hypoalgesia. In addition, it sensitizes subjects to becoming hypoalgesic $24 \mathrm{~h}$ later upon exposure to mild "reinstating" shock. In the present study, we tested whether another manipulation that elicits a hormonally mediated opioid hypoalgesia, food deprivation, would have a similar sensitizing effect. Subjects were food deprived for $48 \mathrm{~h}$, and then, $24 \mathrm{~h}$ after food was returned, they were exposed to mild shock. We found that previously food-deprived subjects exhibited a much stronger hypoalgesia after mild shock. In addition, they exhibited greater vocalization and struggling when shock was applied. Both effects were attenuated by the opiate antagonist naltrexone. In contrast to past results, only a weak hypoalgesia was observed in the saline-treated nondeprived subjects. Experiment 2 showed that this was due to our having tested subjects during the early portion of the light cycle.
\end{abstract}

Considerable evidence now exists that exposure to a variety of aversive stimuli (e.g., shock, immobilization, and cold water) can produce a strong decrease in pain reactivity, or hypoalgesia (Akil, Madden, Patrick, \& Barchas, 1976; Amir \& Amit, 1978; Bodnar, Kelly, Brutus, \& Glusman, 1980). In previous experiments, we have studied this phenomenon by exposing rats to the uncontrollable shock procedure used to induce behavioral learned helplessness (Grau, Hyson, Maier, Madden, \& Barchas, 1981; Hyson, Ashcraft, Drugan, Grau, \& Maier, 1982; Maier et al., 1980). We found that this shock schedule produces a hypoalgesia that is opioid in form, because it is blocked by both opiate antagonists (i.e., naloxone and naltrexone) and morphine tolerance (Drugan, Grau, Maier, Madden, \& Barchas, 1981; Grau et al., 1981; Lewis, Cannon, \& Liebeskind, 1980; Maier et al., 1980). Unlike the opioid hypoalgesia observed in most situations (Fanselow, 1984; Terman, Shavit, Lewis, Cannon, \& Liebeskind, 1984; Watkins \& Mayer, 1982), the hypoalgesia observed after an extended exposure to inescapable shock appears to be "hormonally" mediated, since it is blocked by manipulations that disrupt the pituitary-adrenal axis (e.g., adrenalectomy, hypophysectomy, and administration of dexamethasone) (Lewis et al., 1980; Lewis, Tordoff, Sherman, \& Liebeskind, 1982; MacLennan et al., 1982; Maier, 1986, 1989; Watkins \& Mayer, 1982).

Work on this manuscript was supported by grants to J. W. Grau from the National Institute of Drug Abuse (DA05846-01), the National Science Foundation (BNS-881981), and the Texas Advanced Research Program (010366-09). The authors would like to thank Mary Meagher and Cathy Grover for their comments on this paper. Correspondence concerning this article should be addressed to Paul A. Illich, Department of Psychology, Texas A\&M University, College Station, TX 77843.
Exposure to an extended series of inescapable shocks also has long-term consequences. For example, it sensitizes subjects to becoming hypoalgesic, $24 \mathrm{~h}$ later, upon exposure to mild "reinstating" shock (see, e.g., Grau et al., 1981; Maier, 1986; Maier et al., 1980). This "longterm"' hypoalgesia also appears to be mediated by a hormonal opioid system, because it is blocked by naltrexone, morphine tolerance, dexamethasone, adrenalectomy, and hypophysectomy (Drugan et al., 1981; Grau et al., 1981; MacLennan et al., 1982; Maier et al., 1980). Importantly, this effect does not reflect either a conditioned or a residual hypoalgesia, because previously inescapably shocked rats do not exhibit any hypoalgesia in the absence of the reinstating shock (Jackson, Maier, \& Coon, 1979). These findings led Grau and his colleagues to suggest that prior exposure to inescapable shock "sensitizes" the opioid system (Grau, 1987a; Grau et al., 1981).

Because the opioid system is not sensitized if naltrexone or dexamethasone are given prior to the initial exposure to inescapable shock (MacLennan et al., 1982; Maier et al., 1980), it has been suggested that the activation of the hormonal opioid system plays a critical role in producing the sensitization effect (Grau, 1987a). Given this, one might wonder whether other manipulations that activate the hormonal system have a similar sensitizing effect. For example, considerable evidence exists that food deprivation elicits a strong hormonally mediated opioid hypoalgesia (Hamm \& Knisely, 1986; Hamm, Knisely, Watson, Lyeth, \& Bossut, 1985; McGivern, Berka, Berntson, Walker, \& Sandman, 1979; McGivern \& Berntson, 1980). If activation of the hormonal opioid system is sufficient to sensitize the opioid system, then previously fooddeprived subjects should exhibit a heightened opioid hypoalgesia upon exposure to mild shock. Experiment 1 was a test of this hypothesis. 


\section{EXPERIMENT 1}

To test whether previously food-deprived subjects exhibit a heightened opioid hypoalgesia upon exposure to mild shock, we deprived half of the subjects of food for $48 \mathrm{~h}$. Food was then returned, and $24 \mathrm{~h}$ later, the subjects were exposed to three mild shocks $(0.75 \mathrm{sec}, 1 \mathrm{~mA})$. Elsewhere, we have shown that this shock schedule elicits both a transient, naltrexone-insensitive "nonopioid" and a longlasting, naltrexone-reversible opioid hypoalgesia (for evidence that these effects do not reflect either a locally mediated hypoalgesia or a disruption of motor reactivity, see Illich \& Grau, 1990). To assess the impact of food deprivation on these two components, half of the subjects in each condition were given naltrexone prior to testing.

If food deprivation sensitizes subjects to becoming hypoalgesic, then previously food-deprived subjects should exhibit a much stronger hypoalgesia after mild shock. However, one could argue that this potentiated hypoalgesia simply reflects a residual food-deprivation-induced hypoalgesia that summates with the hypoalgesia elicited by mild shock. To rule out this alternative, we must show that previously food-deprived subjects do not exhibit any residual hypoalgesia $24 \mathrm{~h}$ after food is returned. In the present experiment, we addressed this issue in a number of ways. First, baseline levels of pain reactivity were assessed with the tailflick test immediately before mild shock was administered. In addition, unshocked controls were used to verify that no differences were observed between groups in the absence of shock. Finally, we assessed the impact of food deprivation on the vocalization and struggling elicited by the mild shock used to induce hypoalgesia.

\section{Method}

Subjects. The subjects were 48 male Sprague-Dawley rats obtained from Harlan (Houston, TX). The rats were 100-120 days old and weighted $420-480 \mathrm{~g}$. They were maintained on a 12:12-h light:dark cycle. The light phase began at 6:30 am and ended at $6: 30 \mathrm{pm}$. Testing was conducted during the first $3 \mathrm{~h}$ of the light phase of the light:dark cycle. The subjects were individually housed and had food and water continuously available.

Apparatus. The apparatus used to restrain, to shock, and to test pain reactivity has been described in detail elsewhere (Grau, 1987a, $1987 \mathrm{~b}$ ). Here it will be described briefly. During behavioral testing, the rats were restrained in one of two Plexiglas tubes $(22 \mathrm{~cm}$ in length and $6.8 \mathrm{~cm}$ in diameter). A $660-\mathrm{V}$ transformer was used to produce a constant-current $1.0-\mathrm{mA}$ shock. The shock was administered through tail electrodes, which were constructed from a modified fuse clip. The electrode was taped to the rat's tail, approximately $15 \mathrm{~cm}$ behind the rear of the tubes. Tailflick latencies were assessed with a radiant heat tailflick device.

Vocalization was measured with a miniature microphone (Radio Shack 270-092B), which was positioned over a 9.4- $\mathrm{mm}$ hole drilled through the top of each tube $2.5 \mathrm{~cm}$ from the front. The microphone was connected to a Sanyo amplifier (DCA 611), which was adjusted to selectively amplify frequencies above $1500 \mathrm{~Hz}$. At $80 \mathrm{~dB}$, frequencies below $1500 \mathrm{~Hz}$ were attenuated by approximately $8 \mathrm{~dB}$. The response function of the system was relatively flat $( \pm 0.5 \mathrm{~dB})$ from 1500 to $20000 \mathrm{~Hz}$. The output from the amplifier was fed into a full wave rectifier. This provided a dc voltage that was proportional to the intensity of the sound pressure level at the microphone. The dc voltage was fed into an analog-to-digital converter (Alpha Products, Analog 80), which was connected to a Radio Shack Model IV computer. The computer read and recorded the digital input approximately 19 times per second. This system has been calibrated by determining the relation between the digital input and the loudness of a $4000-\mathrm{Hz}$ sine-wave tone. The computer used this derived function to convert each of the digital inputs to decibels. Sounds below $78 \mathrm{~dB}$ were ignored. This cutoff helped to prevent extraneous sounds, such as breathing, from contaminating our vocalization data. The system could measure sounds up to an intensity of $110 \mathrm{~dB}$.

Struggling was measured by attaching a strain gauge to the tail electrodes. The strain gauge was connected to the electrodes by a wire and was positioned $20 \mathrm{~cm}$ behind the electrodes. The strain gauge was constructed from a spring-loaded potentiometer. This potentiometer was connected to an adjustable voltage regulator that controlled the voltage received by the analog-to-digital converter. The output from each of the strain gauges was sampled approximately 19 times per second. This system has been calibrated by computing the relation between the digital inputs and force in newtons $(N)$. The system could measure forces up to $10 \mathrm{~N}$.

Procedure. The experiment was conducted in three phases: (1) baseline testing; (2) an assessment of the impact of food deprivation, per se, on pain reactivity; and (3) a test of the long-term consequences of food deprivation on pain reactivity and shockinduced hypoalgesia. During the first phase of baseline testing (Days 1 and 2), subjects were placed in restraining tubes and allowed $20 \mathrm{~min}$ to become acclimated. They were then given four tailflick tests spaced 2 min apart. An 8-sec cutoff was used to prevent tissue damage. The average of the last three tailflick latencies on Day 2 was used as the baseline measure of pain reactivity. Immediately after testing was completed on Day 2, subjects were placed in one of two groups (food-deprivation phase). For one group (deprived), food was removed. The impact of food deprivation on pain reactivity was tested $24 \mathrm{~h}$ later (Day 3). This test was conducted in the same manner as has been described for Days 1 and 2. After this test, subjects were given $9 \mathrm{~g}$ of food. Pain reactivity was assessed again $24 \mathrm{~h}$ later (Day 4). At the end of testing, all of the fooddeprived subjects were maintained on ad lib food for the remainder of the experiment. The remaining subjects (nondeprived) were allowed free access to food throughout the experiment. These subjects were tested in the same manner as were the deprived subjects on Days 3 and 4.

On Day 5, we tested the long-term consequences of food deprivation. Prior to testing, half the subjects in each condition received an i.p. injection of naltrexone $(14 \mathrm{mg} / \mathrm{kg})$. The remaining subjects received an injection of saline. Ten minutes later, subjects were placed in restraining tubes and given $\mathbf{2 0}$ min to become acclimated. The subjects then received four tailflick tests spaced 2 min apart. Immediately after the last tailflick test, shock electrodes were attached and half of the subjects in each condition received three 0.75 sec, 1-mA tailshocks at 20-sec intervals. Vocalization and struggling were recorded in the shocked subjects for $1 \mathrm{sec}$ before, $1 \mathrm{sec}$ during, and $5 \mathrm{sec}$ after each shock. Two minutes after the last shock or an equivalent period of restraint, all subjects received five tailflick tests spaced 2 min apart.

Statistics. The preshock vocalization and straining results were analyzed with the nonparametric Kruskal-Wallis $H$ test. All other data were analyzed with an analysis of variance (ANOVA). Post hoc comparisons were made with the Duncan's multiple-range test.

\section{Results}

Day 2: Tailflick scores. The test of baseline levels of pain reactivity on Day 2 revealed that no differences existed prior to testing $[F(1,46)=0.04, p>.05]$. The mean tailflick latencies were 4.84 and $4.86 \mathrm{sec}$ for the food-deprived and -nondeprived groups, respectively. 
Days 3 and 4: Tailfick scores. To assess the impact of food deprivation on tailflick latencies, we computed the change from baseline scores by subtracting each subject's baseline score obtained on Day 2 from the mean tailflick latencies observed on Days 3 and 4 . These values are shown in Figure 1. Although it appears that food deprivation induced a slight hypoalgesia, this effect did not approach statistical significance (all $F \mathrm{~s}<2.54, p>.05$ ).

Day 5: Baseline tailflick latencies. The mean tailflick latencies observed immediately before either mild shock or an equivalent period of restraint (unshocked) are depicted in Figure 2. Although there appeared to be some variation among groups, an ANOVA revealed that these differences did not reach statistical significance (all $F \mathrm{~s}<$ $3.55, p>.05$ ).

Day 5: Vocalization. A detailed analysis of the vocalization data revealed that only 4 of the 24 shocked subjects ( 2 of the naltrexone nondeprived subjects and 2 of the saline deprived subjects) vocalized prior to shock. The small difference that this produced between the overall means $(0.9 \mathrm{~dB})$ was not significant [Kruskal-Wallis $H(3)=4.57$, $p>.05]$.

The levels of vocalization observed during each shock are depicted in the top panels of Figure 3. It appears that previously food-deprived subjects vocalized more than nondeprived control subjects did, and that this effect was attenuated by naltrexone. An ANOVA revealed that the main effect of food deprivation was significant $[F(1,20)$ $=7.02, p<.05$ ]. However, neither the main effect of drug treatment $[F(1,20)=2.72, p>.05]$ nor its interaction with deprivation condition $[F(1,20)=1.41, p>$ $.05]$ was statistically significant. Also, none of the trials effects were significant (all $F \mathrm{~s}<2.41, p>.05$ ). Post hoc comparisons showed that previously food-deprived subjects that received saline vocalized more than did both nondeprived groups. No other comparisons were significant.

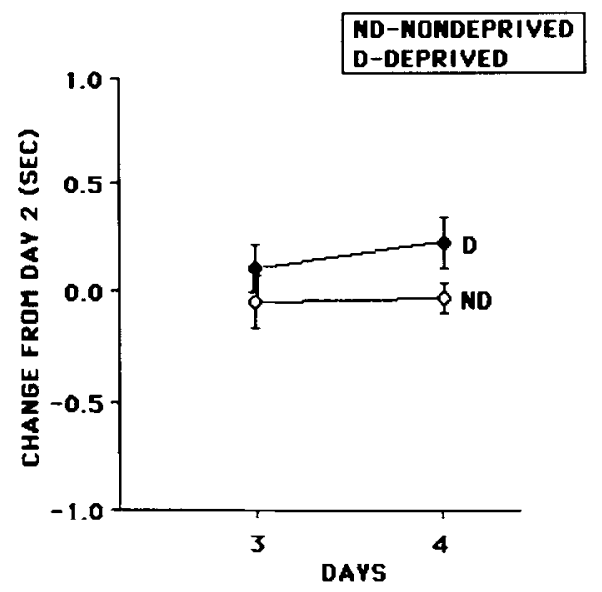

Figure 1. Impact of 24 h (Day 3) and 48 h (Day 4) of food deprivation. Subjects were either food deprived (filled circles) or maintained on ad-lib food (open circles). The error bars represent standard error of the mean.

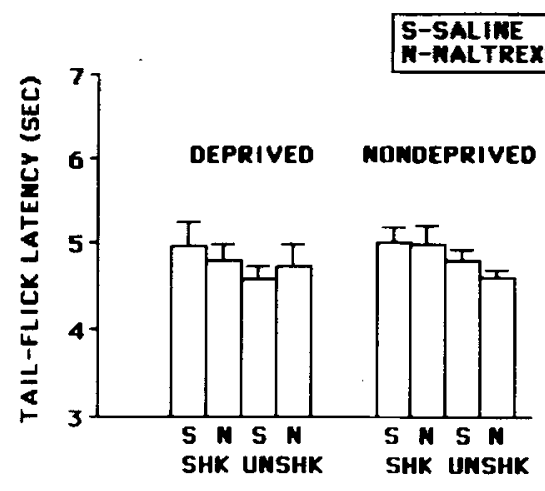

Figure 2. Baseline levels of pain reactivity observed in subjects that were subsequently shocked (SHK) or remained unshocked (UNSHK). Subjects that had been previously food deprived (DEPRIVED) are shown in the left portion of the figure, while subjects that had continuous access to food (NONDEPRIVED) are depicted on the right. Half of the subjects in each condition were injected with either saline (S) or naltrexone (N). The error bars represent standard errors of the mean.

After shock (see bottom panels of Figure 3) it appears that food deprivation again increased the amount of vocalization observed in the saline controls, and that this effect was attenuated by naltrexone. Interestingly, in the nondeprived subjects, naltrexone seems to have had the opposite effect on pain reactivity, increasing the vocalization observed after shock. An ANOVA confirmed that the interaction between food deprivation and drug treatment was significant $[F(1,20)=9.48, p<.01]$. Neither the main effect of food deprivation $[F(1,20)=0.45$, $p>.05]$ nor that of drug treatment $[F(1,20)=0.20$, $p>.05$ ] approached statistical significance. None of the trials effects were significant (all $F s<1.96, p\rangle .05$ ). Post hoc comparisons showed that previously fooddeprived subjects that received saline vocalized more than did nondeprived saline subjects and deprived naltrexone subjects. No other comparisons were significant.

Day 5: Straining. Prior to shock treatment, the mean levels of straining were $0.75,0.15,0.30$, and $0.11 \mathrm{~N}$ for the saline nondeprived, naltrexone nondeprived, saline deprived, and naltrexone deprived groups, respectively. These between-group differences were significant [KruskalWallis $H(3)=10.56, p<.01]$. To compensate for these baseline differences, we subtracted each subject's baseline score from the level of straining that it exhibited during and after shock.

The levels of straining observed during shock are depicted in the top panels of Figure 4. It again appears that previously food-deprived subjects exhibited heightened reactivity to shock, and that this effect was reversed by naltrexone. An ANOVA confirmed that both the main effect of food deprivation $[F(1,20)=6.47, p<.05]$ and its interaction with drug treatment $[F(1,20)=8.26, p<$ $.01]$ were significant. The main effect of drug treatment $[F(1,20)=2.61, p>.05]$, and all of the trials effects 


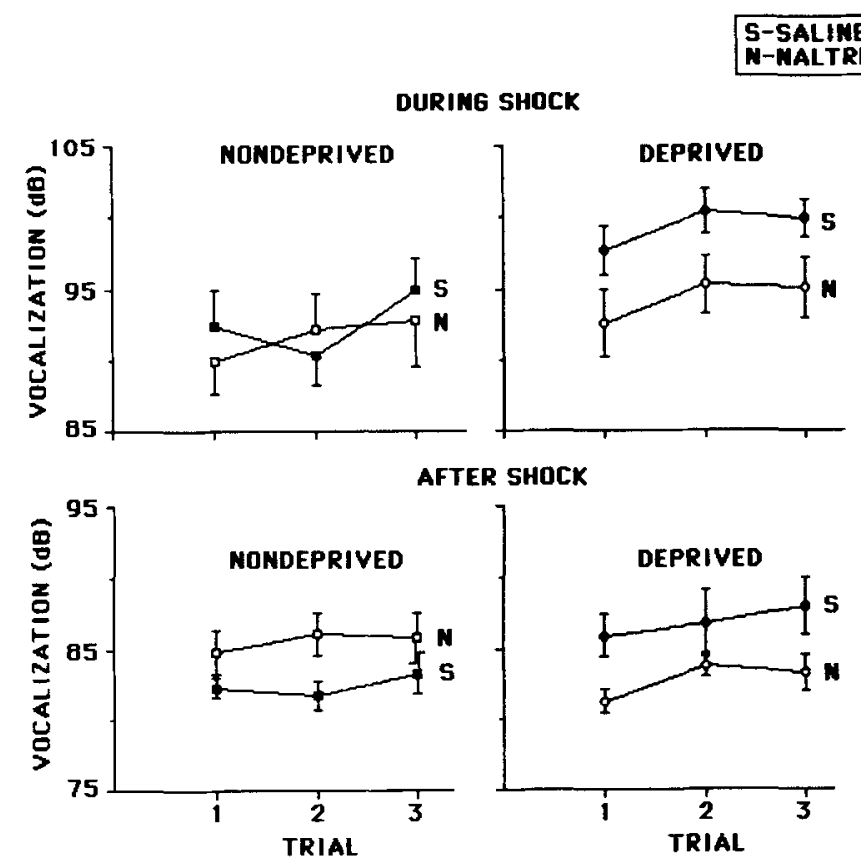

Figure 3. Magnitude of vocalization during (upper panels) and after (lower panels) shock in saline- (filled symbols) and naltrexone- (open symbols) treated subjects that had been previously food deprived (right panels) or nondeprived (left panels). The error bars indicate standard error of the mean.
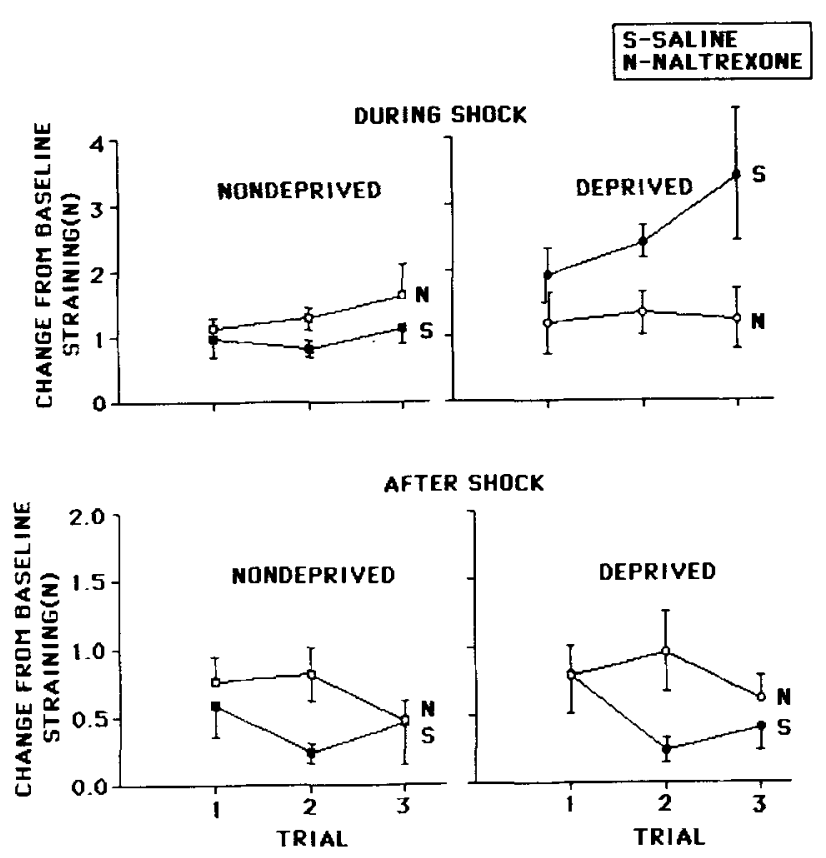

Figure 4. Change from pre-straining scores observed during (upper panels) and after (lower panels) shock in saline- (filled symbols) and naltrexone- (open symbols) treated subjects that had been previously food deprived (right panels) or nondeprived (left panels). The error bars indicate standard error of the mean. (all $F_{\mathrm{S}}<2.93, p>.05$ ), failed to reach statistical significance. Post hoc comparisons revealed that subjects in the saline-treated food-deprived group strained significantly more than did subjects in the other three groups. No other comparisons were significant.

It appears that naltrexone-treated subjects strained slightly more after shock than did the saline controls (see Figure 4, bottom panel). An ANOVA confirmed that the main effect of drug treatment was significant $[F(1,20)=$ $4.76, p<.05]$. Neither the main effect of food deprivation $[F(1,20)=0.43, p>.05]$ nor its interaction with drug treatment $[F(1,20)=0.06, p>.05]$ approached significance. None of the trials effects were statistically significant (all $F \mathrm{~s}<2.81, p>.05$ ].

Day 5: Postshock tailflick latencies. The levels of pain reactivity observed on the tailflick test after shock, or an equivalent period of restraint, are depicted in Figure 5. To control for the variability observed in tailflick latencies prior to shock, a change from baseline score was employed. This was computed by simply subtracting each subject's baseline score observed on Day 5 from the tailflick latencies observed after shocked subjects received shock. Inspection of the adjusted scores reveals that exposure to mild shock elicited a transient hypoalgesia (top left) that was eliminated by naltrexone (bottom left). In contrast, saline-treated subjects that had been previously food deprived exhibited a robust hypoalgesia after mild 


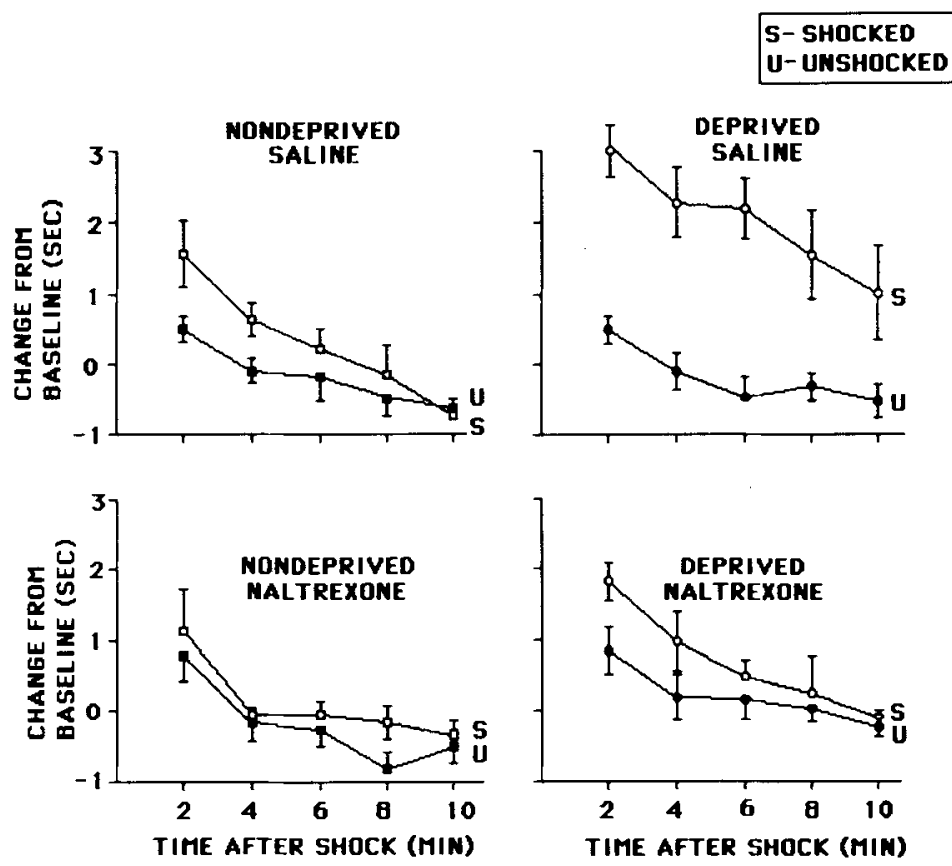

Figure 5. Change from baseline tailnick latencies for saline- (upper panels) and naltrexone- (lower panels) treated subjects that received shock (open symbols) or remained unshocked (closed symbols). Half of the subjects had been previously food deprived (right panels), while the other half had food continuously available (left panels). The error bars indicate standard error of the mean.

shock (top right), and this hypoalgesia was attenuated by naltrexone (bottom right). An ANOVA confirmed these impressions. Because the between-subject manipulations did not interact with any of the trials effects (all $F \mathrm{~s}<$ $2.12, p>.05$ ), the data were collapsed across the five postshock trials. Both the main effect of shock $[F(1,40)$ $=40.11, p<.001]$ and the main effect of food deprivation $[F(1,40)=23.77, p<.001]$ were significant. More importantly, the interaction between shock and food deprivation $[F(1,40)=12.40, p<.01]$ and the threeway interaction between shock, food deprivation, and drug treatment $[F(1,40)=7.92, p<.01]$ were statistically significant. In addition, the main effect of drug treatment $[F(1,40)=4.57, p<.05]$ and its interaction with shock $[F(1,40)=12.40, p<.01]$ were significant. The interaction between food deprivation and drug treatment did not approach statistical significance $[F(1,40)=1.96$, $p>.05]$. Post hoc comparisons confirmed that previously food-deprived subjects that received saline and shock were hypoalgesic relative to all other subjects. In addition, previously food-deprived subjects that received shock and naltrexone were hypoalgesic relative to all of the unshocked groups, except the one that had been previously food deprived and received naltrexone. No other comparisons were significant.

A point that remains unclear from the preceding analyses is whether food deprivation potentiated the nonopioid hypoalgesia we normally observe 2-4 min after mild shock (Grau, 1984). To address this issue, a separate anal- ysis was conducted on the mean level of pain reactivity observed 2-4 min after shock (as before, the changes from baseline scores were analyzed). This analysis revealed that the main effects of both food deprivation $[F(1,40)=$ $12.44, p<.01]$ and shock treatment $[F(1,40)=31.24$, $p<.001]$ were statistically significant. Most importantly, the impact of shock treatment depended both on drug treatment $[F(1,40)=8.11, p<.01]$ and on whether or not the subjects had been previously food deprived $[F(1,40)$ $=7.98, p<.01]$. The main effect of drug treatment was not significant $[F(1,40)=3.71, p>.05]$. None of the other interactions approached statistical significance (both $F_{\mathrm{s}}\langle 1.39, p\rangle .05$ ). Post hoc comparisons showed that the saline-treated shocked subjects that had been previously food deprived were hypoalgesic relative to all other groups. The naltrexone-treated shocked subjects that were food deprived were hypoalgesic relative to all groups except the nondeprived saline-treated shocked subjects. In addition, the nondeprived saline shocked group was hypoalgesic relative to the saline-treated nondeprived unshocked group. No other differences were significant.

\section{Discussion}

We found that during the period when subjects were food deprived, food deprivation had little impact on pain reactivity as measured by the tailflick test. This was an unexpected finding, because others have routinely reported that a similar deprivation schedule induces a robust hypoalgesia on this test (e.g., Hamm \& Knisely, 1986; Hamm 
et al., 1985). Recent evidence from our laboratory suggests that our failure to observe an effect of food deprivation was due to our having tested during the early portion of the light cycle, a time at which a number of hypoalgesic effects are at their minimum (e.g., conditioned hypoalgesia, morphine hypoalgesia) (Frederickson, Burgis, \& Edwards, 1977; Illich \& Grau, in press). Specifically, we found that a strong hypoalgesia is only observed in food-deprived subjects when they are tested during the early portion of the dark cycle (Biles, Illich, \& Grau, in press).

As predicted, food deprivation potentiated the hypoalgesia observed after mild shock. This supports the hypothesis that activation of the hormonal opioid system is sufficient to sensitize subjects to becoming hypoalgesic. As in studies in which inescapable shock was used to induce the sensitization effect (e.g., Grau et al., 1981; Maier, 1986), we found that the potentiated hypoalgesia was attenuated by the opiate antagonist naltrexone. In contrast with the results of these past studies, we also found that previously stressed subjects exhibited a stronger naltrexone-insensitive, nonopioid hypoalgesia. This difference in the pattern of results may be attributed to a basic difference in how subjects were treated. In the present experiment, the subjects remained undisturbed in the test chamber throughout testing, whereas in studies in which inescapable shock was used to induce the sensitization effect, testing required removing the subjects from the chamber in which the reinstating shock was experienced. Removing the subjects in this fashion would effectively expose them to a variety of distracting stimuli. Elsewhere, we have shown that distracting the subjects immediately after they are exposed to mild shock acts to attenuate the transient nonopioid hypoalgesia, replacing it with a hypoalgesia that is naltrexone reversible. Thus, the experimental design in the present experiment may have allowed us to observe a potentiated nonopioid hypoalgesia by minimizing the degree to which subjects were distracted during testing.

From our tests of baseline levels of pain reactivity, it is clear that the potentiated hypoalgesia observed after mild shock does not reflect the summation of a residual food-deprivation-induced hypoalgesia with the hypoalgesia elicited by mild shock. In support of this, we failed to observe any differences between the nondeprived and deprived subjects on the spinally mediated tailflick test prior to shock. Moreover, after shock was applied to half the subjects, no differences were observed in the unshocked controls. Most importantly, previously fooddeprived subjects were not less reactive to the shock used to induce hypoalgesia. In fact, the opposite was observed; a history of food deprivation potentiated both shockinduced vocalization and struggling. Thus, instead of a residual hypoalgesia, hyperalgesia was observed. Interestingly, this hyperalgesia appears to be opioid mediated, because it was attenuated by naltrexone. A similar effect has been observed with other paradigms (Davis \& Hendersen, 1985), and we will discuss the relation of this work to our own in more detail in the General Discussion. For the present, we would like simply to note that this opioidmediated hyperalgesia may provide an explanation for why a history of stress sensitizes subjects to becoming hypoalgesic. Specifically, it suggests that a greater hypoalgesia may be observed because previously stressed rats perceive the shock as more aversive. Moreover, our results suggest a very different interpretation of how naltrexone affects the potentiated hypoalgesia observed in previously stressed subjects. Until now, it has been assumed that naltrexone attenuates the potentiated hypoalgesia because it blocks the action of the opioids released by the mild reinstating shock. From this perspective, a history of uncontrollable stress potentiates the hypoalgesia observed after mild shock because it increases the amount of opioids released (Grau, 1987a; Maier, 1986). However, our results suggest that a history of stress may affect shock-induced hypoalgesia in a very different way, by producing an opioid-mediated increase in the perceived aversiveness of the shock. Thus, naltrexone may attenuate the magnitude of the hypoalgesia observed in previously stressed rats simply because it prevents stress from altering the hedonic value of the shock. From this perspective, it is not necessary to assume that the hypoalgesia observed after mild shock is produced by the release of endogenous opioids. Instead, the potentiated hypoalgesia observed after shock could be mediated by a naltrexoneinsensitive, nonopioid system. If this is true, then administering naltrexone after shock is applied should have no impact on the magnitude of hypoalgesia observed. Interestingly, others have reported that some hypoalgesic effects can be prevented, but not reversed, by administration of an opioid antagonist (Watkins \& Mayer, 1982).

\section{EXPERIMENT 2}

The results of Experiment 1 demonstrated that previously food-deprived subjects exhibit an exaggerated hypoalgesia $24 \mathrm{~h}$ later upon exposure to mild shock. Surprisingly, we found that mild shock per se produced only a small hypoalgesia in the saline-treated nondeprived subjects, and that this effect was only statistically significant over the period from 2 to 4 min after shock. Although a separate analysis of the mean tailflick latencies observed 2-10 min after shock in saline-treated nondeprived controls did reveal a significant overall difference $[F(1,10)$ $=5.01, p<.05]$, it is clear that the magnitude of the hypoalgesia observed was much smaller than that found in past studies (i.e., Grau, 1984, 1987a). Moreover, in contrast to the results of past work, the small hypoalgesia that was observed in the nondeprived subjects from 2 to 4 min postshock appeared to be opioid mediated. These observations suggest that a novel aspect of our testing procedure acted to attenuate both the nonopioid and the opioid hypoalgesia normally observed after mild shock.

There are a number reasons why mild shock may have had such a small effect on pain reactivity in Experiment 1. One is that subjects had previously received 4 days of 
preexposure to the test environment. This preexposure may attenuate shock-induced hypoalgesia because it undermines the association of shock to the test context. Indeed, there is a great deal of evidence that preexposure of a conditioned stimulus undermines Pavlovian conditioning. Given that some (Fanselow, 1984; Grau, 1987a; Maier, 1989) have suggested that mild-shock-induced hypoalgesia may, at least in part, be a conditioned effect, one would expect preexposure to the experimental context to undermine the development of mild-shock-induced hypoalgesia.

A second reason that the nondeprived subjects may have exhibited little shock-induced hypoalgesia is that testing was conducted during a different portion of the light:dark cycle; in Experiment 1, we tested during the early portion of the light cycle, whereas in all of our previous studies, we tested subjects during either the late portion of the light cycle or the early part of the dark cycle. This difference may be critical, since a variety of hypoalgesic effects have been reported to be greatest during the dark cycle, and smallest during the light cycle (Frederickson et al., 1977; Illich \& Grau, in press).

In Experiment 2, we assessed whether our failure to observe mild-shock-induced hypoalgesia in Experiment 1 was due to either preexposure to the experimental context or the time of day at which subjects were tested.

\section{Method}

Subjects. The subjects were 48 rats of the same sex, age, and weight used in Experiment 1.

Apparatus. The apparatus was the same as was used in Experiment 1 .

Procedure. One third of the subjects (preexposed, early light) received 4 days of tailflick testing as described in Experiment 1. On Day 5, half of these subjects received three 0.75-sec, 1-mA tailshocks spaced $20 \mathrm{sec}$ apart. They then received five tailflick tests at 2-min intervals. The other half were treated similarly, except that shock was omitted. For both preexposure groups, testing was conducted during the first $3 \mathrm{~h}$ of the light cycle. Another third (nonpreexposed, early light) were treated the same as were the preexposed subjects, with the exception that the 4 days of preexposure were omitted. The final third (nonpreexposed, late light) were treated as were the nonpreexposed, early light subjects, except that testing occurred during the last $3 \mathrm{~h}$ of the light cycle.

Statistics. The data were analyzed as in Experiment 1.

\section{Results}

The results from subjects tested during the early portion of the light cycle are illustrated in Figure 6. It is clear that prior exposure to the test context did not affect baseline levels of pain reactivity (all $F \mathrm{~s}<0.97, p>.05$ ). Preexposure also had little impact on the magnitude of mild-shock-induced hypoalgesia. Because the trials effect did not interact significantly with drug treatment (all $F \mathrm{~s}\langle 1.57, p\rangle .05$ ), the data were collapsed across the five postshock test trials. An analysis of these means revealed that shock had a significant impact on pain reactivity $[F(1,28)=20.60, p<.01]$. Neither the impact of preexposure $[F(1,28)=0.93, p>.05]$ nor its interaction with shock $[F(1,28)=0.19, p>.05]$ was statistically significant. Post hoc comparisons confirmed that both the preexposed and the nonpreexposed shocked groups

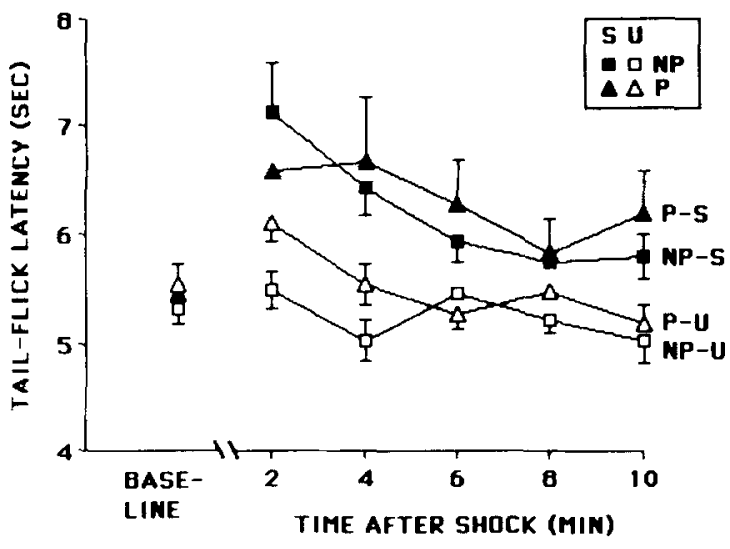

Figure 6. Tailnick latencies observed in subjects that had been preexposed (P) or nonpreexposed (NP) to the test context. Subjects that received mild shock $(S)$ or remained unshocked $(U)$ are depicted by filled and open symbols, respectively. The error bars indicate standard error of the mean.

were hypoalgesic relative to the unshocked controls. No other comparisons were significant.

The results from subjects that were tested during the late portion of the light cycle are depicted in Figure 7. For purposes of comparison, the data from the nonpreexposed subjects that were tested during the early portion of the light cycle are also presented. It is apparent that the time of testing had little impact on baseline levels of pain reactivity (all $\left.F_{\mathrm{S}}<0.55, p\right\rangle .05$ ). After shock, subjects exhibited a stronger hypoalgesia when they were tested during the late portion of the light cycle. An ANOVA confirmed that both the main effect of shock treatment $[F(1,28)=58.66, p<.01]$ and its interaction with time of testing $[F(1,28)=4.43, p<.05]$ were significant. Moreover, the magnitude of this interaction depended on the trial of testing $[F(4,112)=2.57, p<.05]$. The only other term that was statistically significant was the main effect of test trial $[F(4,112)=9.62, p<.01]$.

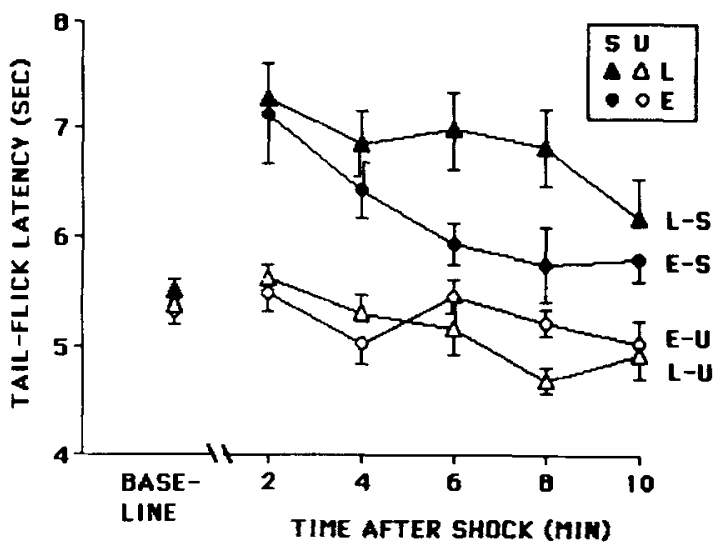

Figure 7. Tailflick latencies observed in subjects tested during either the early $(E)$ or the late $(L)$ portion of the light cycle. Subjects that received shock (S) or remained unshocked (U) are depicted by filled and open symbols, respectively. The error bars indicate standard error of the mean. 
Post hoc comparisons of the overall means showed that shocked subjects that were tested during the late portion of the light phase were hypoalgesic relative to all other subjects. Subjects that received shock and were tested during the early part of the light phase were hypoalgesic relative to the two unshocked groups. No other comparisons were significant.

\section{Discussion}

As in Experiment 1, a small but statistically significant hypoalgesia was observed after mild shock in the preexposed subjects. Surprisingly, nonpreexposed subjects tested during the same early portion of the light cycle exhibited a similarly small shock-induced hypoalgesia. Thus, the hypoalgesia observed was not affected by preexposure to the restraining tubes. Regarding this failure, a couple of points should be noted. First, our primary concern was the failure to observe a robust shock-induced hypoalgesia in the nondeprived subjects of Experiment 1 . To address this issue, we contrasted the procedure used in that experiment with that typically used in our laboratory. Thus, it is quite possible that other preexposure manipulations would affect the magnitude of mild-shock-induced hypoalgesia. In fact, we have recently shown that removing the 20-min preexposure that subjects experience prior to testing profoundly affects the magnitude of the hypoalgesia observed. However, instead of attenuating the hypoalgesia, we found that prior exposure to the test apparatus potentiated mild-shock-induced hypoalgesia (Biles, 1991).

As in past studies (Grau, 1984, 1987a, 1987b), a robust hypoalgesia was observed when subjects were tested during the late portion of the light cycle. This finding, in conjunction with the results of Experiment 1, suggests that there are diurnal fluctuations in the magnitude of both the nonopioid and opioid hypoalgesia observed after mild shock; relative to the early portion of the light cycle, these hypoalgesic responses are much stronger when subjects are tested during the late portion of the light cycle. Elsewhere (Grau, 1987a, 1987b; Grau, Illich, Chen, \& Meagher, 1991), we have shown that manipulations that disrupt memory (e.g., presentation of a postshock distractor) have a similar affect on the form and duration of mild-shockinduced hypoalgesia. Specifically, these manipulations cause both the nonopioid and opioid hypoalgesia to decrease much more rapidly. The consequence of this is that, at most, only a transient hypoalgesia is observed 2-4 $\mathrm{min}$ after shock, and this hypoalgesia is naltrexone reversible. Given that testing during the early portion of the light cycle has an identical impact on mild-shock-induced hypoalgesia, it is tempting to speculate that this effect too is due to variation in learning and memory. Specifically, nocturnal rats may show little mild-shock-induced hypoalgesia in the early portion of the light cycle because this is the time at which learning and memory are poorest.

\section{GENERAL DISCUSSION}

The present experiments examined whether previously food-deprived subjects, like previously inescapably shocked subjects, would exhibit a heightened opioid hypoalgesia after mild shock. Experiment 1 showed that food deprivation had little impact on tailflick latencies, both during the period when food was removed and $24 \mathrm{~h}$ after food was returned. The fact that subjects did not exhibit any hypoalgesia during the time when they were food deprived contrasts with other reports that food deprivation per se elicits a robust hypoalgesia. However, in Experiment 1 we tested subjects during the early portion of the light cycle, when it is known that a number of other hypoalgesic effects are at their minimum. Given this, we suggested that fooddeprivation-induced hypoalgesia might exhibit a diurnal rhythm, being smallest during the early portion of the light cycle and largest during the early portion of the dark cycle. In support of this, we have shown that the present fooddeprivation schedule does induce hypoalgesia, but that this effect is only observed during the early part of the dark cycle (Biles et al., in press).

Twenty-four hours after food was returned, subjects that had been food deprived were not different from their nondeprived controls, as assessed by the spinally mediated tailflick test. In contrast, our supraspinal measure of pain reactivity, shock-induced vocalization and straining, revealed that previously food-deprived subjects were more reactive during and following shock. These findings suggest that food deprivation increased the perceived aversiveness of the shock and/or its affective impact. Interestingly, this effect was attenuated by naltrexone, which suggests that the hyperalgesia is opioid mediated.

After mild shock, previously food-deprived subjects exhibited a much stronger hypoalgesia. This potentiated hypoalgesia appears to have a large opioid component, because it was attenuated by naltrexone. In addition, previously food-deprived subjects exhibited a significantly greater naltrexone-insensitive, nonopioid hypoalgesia. Thus, the pattern of results observed in previously food-deprived subjects was slightly different from that observed after inescapable shock; food deprivation potentiated both the opioid and the nonopioid components, whereas prior exposure to inescapable shock only appears to potentiate the opioid component. As discussed above, this discrepancy may be attributed to differences in the way that subjects were tested; in the present study, care was taken to avoid distracting subjects during testing, whereas in studies done with inescapable shock, the subjects were routinely removed from the test chambers during testing (Grau et al., 1981; Hyson et al., 1982; Maier, 1986). This difference may be critical, because evidence suggests that distracting subjects immediately after mild shock eliminates the nonopioid hypoalgesia (Grau, 1987a, 1987b). Thus, prior studies may have failed to observe an effect of inescapable shock on the nonopioid hypoalgesia simply because subjects were distracted prior to testing.

The present results suggest that our prior work has considerable generality, and that a variety of manipulations that activate the hormonal form of opioid hypoalgesia may sensitize subjects to becoming hypoalgesic upon exposure to mild shock. The present study also provides a potential explanation for why stressors that activate the hor- 
monal form of opioid hypoalgesia sensitize subjects to becoming hypoalgesic. At the psychological level, the studies suggest that mild shock elicits greater hypoalgesia because it is perceieved as being more aversive. At the physiological level, the results suggest that this hypoalgesia is mediated by the release of an endogenous opioid. What seems particularly odd about this explanation is that it assumes that a low level of opioid activity can increase reactivity to aversive stimulation instead of attenuating it. Yet it is well known that opioids in general produce hypoalgesia, not hyperalgesia. However, there is some evidence that hypoalgesia is not an inevitable consequence of opioid release. For example, Davis and Hendersen (1985) have shown that a stimulus paired with an aversive event can, under some conditions, elicit hyperalgesia and that this hyperalgesia is blocked by an opiate antagonist. Although it is not clear under what conditions this effect is observed (see Illich, Salinas, \& Grau, 1991), it does suggest that the release of endogenous opiates can sometimes elicit hyperalgesia instead of hypoalgesia. Moreover, van der Kooy and colleagues have suggested that peripherally acting opioids elicit aversion and hyperalgesia, not hypoalgesia (Bechara \& van der Kooy, 1987; van der Kooy, 1986; van der Kooy \& Nagy, 1985).

In the present study, we have shown that a period of mild food deprivation can, like exposure to an extended series of inescapable shocks, sensitize the opioid system. These findings are important not only because they extend the generality of our prior work but also because they provide an alternative paradigm for studying this opioid sensitization effect, one that minimizes the pain and suffering experienced by the experimental subjects. Moreover, the present paradigm would appear to have considerably more ecological validity, because food deprivation, unlike exposure to uncontrollable shock, is a stressor that people do frequently encounter. Further work is needed to verify whether food deprivation and inescapable shock have similar behavioral and physiological consequences.

\section{REFERENCES}

Akal, H., Madden, J., Patrick, R. L., \& Barchas, J. D. (1976). Stressinduced increase in endogenous opiate peptides: Concurrent analgesia and its partial reversal by naloxone. In H. Kosterlitz (Ed.), Opiates and endogenous opioid peptides (pp. 63-70). Amsterdam: Elsevier.

AMIR, S., \& AMIT, Z. (1978). Endogenous opioid ligands may mediate stress-induced changes in the affective properties of pain related behavior in rats. Life Sciences, 23, 1143-1152.

BeCHARA, A., \& VAN DER KOOY, D. (1987). Kappa receptors mediate the peripheral aversive effects of opiates. Pharmacology, Biochemistry \& Behavior, 28, 227-233.

BiLes, M. K. (1991). The role of the pituitary-adrenal axis in mild shockinduced hypoalgesia. Unpublished master's thesis, Texas A\&M University.

Biles, M. K., Illich, P. A., \& Grau, J. W. (in press). Long-term effects of food deprivation: II. Impact on morphine reactivity. Psychobiology.

Bodnar, R. J., Kelly, D. D., Brutus, M., Glusman, M. (1980). Stress-induced analgesia: Neural and hormonal determinants. Neuroscience \& Biobehavioral Reviews, 4, 87-100.
Davis, H., D., \& Hendersen, R. W. (1985). Effects of conditioned fear on responsiveness to pain: Long-term retention and reversibility by naloxone. Behavtoral Neuroscience, 99, 277-289.

Drugan, R. C., Grau, J. W., Maier, S. F., Madden, J., \& Barchas, J. D. (1981). Cross-tolerance between morphine and the long-term reaction to inescapable shock. Pharmacology, Biochemistry \& Behavior, 14, 677-682.

FANSELOW, M. S. (1984). Shock-induced analgesia on the formalin test: Effects of shock severity, naloxone, hypophysectomy, and associative variables. Behavioral Neuroscience, 98, 79-95.

Frederickson, R. C. A., Burgis, V., a Edwards, J. D. (1977). Hyperalgesia induced by naloxone follows diurnal rhythm in responsivity to painful stimuli. Science, 198, 756-758.

GraU, J. W. (1984). The influence of naloxone on shock-induced freezing and analgesia. Behavioral Neuroscience, 98, 278-292.

GRAU, J. W. (1987a). The central representation of an aversive event maintains the opioid and nonopioid forms of analgesia. Behavioral Neuroscience, 101, 272-288.

GrAU, J. W. (1987b). The variables which control the activation of analgesic systems: Evidence for a memory hypothesis and against the coulometric hypothesis. Joumal of Experimental Psychology: Animal Behavior Processes, 13, 215-255.

Grau, J. W., Hyson, R. L., Maier, S. F., Madden, J., Barchas, J. D. (1981). Long-term stress-induced analgesia and activation of the opiate system. Science, 213, 1409-1411.

Grau, J. W., Illuch, P. A., Chen, P.-S., Mragher, M. W. (1991). Role of cholinergic systems in pain modulation: I. Impact of scopolamine on environmentally induced hypoalgesia and pain reactivity. Behavioral Neuroscience, 105, 62-81.

HAMm, R. J., KNISELY, J. S. (1986). The analgesia produced by food deprivation in 4-month old, 14-month old, and 24-month old rats. Life Sciences, 39, 1509-1515.

Hamm, R. J., Knisely, J. S., Watson, A., Lyeth, B. G., Bossut, D. F. B. (1985). Hormonal mediation of the analgesia produced by food deprivation. Physiology \& Behavior, 35, 879-882.

Hyson, R. L., Ashcraft, L. J., Drugan, R. C., Grau, J. W., MAIER, S. F. (1982). Extent and control of shock affects naltrexone sensitivity of stress-induced analgesia and reactivity to morphine. Pharmacology, Biochemistry \& Behavior, 17, 1019-1025.

IlLiCh, P. A., \& GraU, J. W. (1990). The impact of shock on reactivity to a tactile stimulus. Leaming \& Motivation, 21, 287-298.

Illich, P. A., Grau, J. W. (in press). Conditioned changes in pain reactivity: I. A discrete CS elicits hypoalgesia, not hyperalgesia, on the tail-flick test. Learning \& Motivation.

Illich, P. A., Salinas, J. A., \& Grau, J. W. (1991). Conditioned changes in pain reactivity: II. In search of the elusive phenomenon of conditioned hyperalgesia. Behavioral Neuroscience, 105, 478-481.

JACKsON, R. L., MAIER, S. F., COON, D. J. (1979). Long-term analgesic effects of inescapable shock and learned helplessness. Science, 206, 91-93.

Lewis, J. W., Cannon, J. T., \& Liebeskind, J. C. (1980). Opioid and nonopioid mechanisms of stress analgesia. Science, 208, 623-625.

Lewis, J. W., Tordoff, M. G., Sherman, J. E., \& Liebeskind, J. C. (1982). Adrenal medullary enkephalin-like peptides may mediate opioid stress analgesia. Science, 217, 557-559.

Maclennan, J. A., Drugan, R. C., Hyson, R. L., Maier, S. F., MAdDen, J., \& BArChas, J. D. (1982). Corticosterone: A critical factor in an opioid form of stress-induced analgesia. Science, 215 , 1530-1532.

MAIER, S. F. (1986). Stressor controllability and stress-induced analgesia. In D. D. Kelly (Ed.), Stress-induced analgesia (Annals of the New York Academy of Sciences, Vol. 467, pp. 55-72). New York: New York Academy of Sciences.

MAIER, S. F. (1989). Determinants of the nature of environmentally induced hypoalgesia. Behavioral Neuroscience, 103, 131-143.

Maier, S. F., Davies, S., Grau, J. W., Jackson, R. L., Morrison, D. H., Moye, T., Madden, J., \& Barchas, J. D. (1980). Opiate antagonists and long-term analgesic reaction induced by inescapable 
shock in rats. Joumal of Comparative \& Physiological Psychology, 94, 1172-1183.

McGivern, R., Berka, C., Berntson, G. G., Walker, J. M., \& SANDMAN, C. A. (1979). Effect of naloxone on analgesia induced by food deprivation. Life Sciences, 25, 885-888.

McGivern, R., Berntson, G. G. (1980). Mediation by diurnal fluctuations in pain sensitivity in the rat by food intake patterns: Reversal by naloxone. Science, 210, 210-211.

Terman, G. W., Shavit, Y., Lewis, J. W., Cannon, J. T., Liebeskind, J. C. (1984). Intrinsic mechanisms of pain inhibition: Activation by stress. Science, 226, 1270-1277.

VAN DER KOOY, D. (1986). Hyperalgesic functions of peripheral opiate receptors. In D. D. Kelly (Ed.), Stress-induced analgesia (Annals of the New York Academy of Sciences, Vol. 467, pp. 154-168). New York: New York Academy of Sciences.

VAN DER KoOY, D., NAGY, J. I. (1985). Hyperalgesia mediated by peripherial opiate receports in the rat. Behavioral Brain Research, 17, 203-211.

WATKINS, L. R., MAYER, D. J. (1982). Organization of endogenous opiate and nonopiate pain control systems. Science, 216, 1185-1192.

(Manuscript received March 19, 1991; revision accepted for publication August 12, 1991.)

\section{Forthcoming Psychobiology Articles}

The following is a list of forthcoming Psychobiology articles that are currently in press. They are given in approximate order of acceptance. Each entry includes the name and address of the author with whom to communicate for further prepublication information.

"Fluoxetine effects on retention of inhibitory avoidance: Enhancement by systemic but not intra-amygdala injections" by I.B. Introini-Collison, S. To, \& J.L. McGaugh (I.B.I.-C., Ctr. for the Neurobiology of Learning \& Memory, Univ. of California, Irvine, CA 92717)

"Repeated administration of pyrithiamine leads to a proportional increase in the remembered durations of events" by W.H. Meck \& K.E. Angell (W.H.M., Dept. of Psychology, 406 Schermerhorn Hall, Columbia Univ., New York, NY 10027)

"Interaction of the opiate and NMDA systems in a spatial learning task'” by E. Bostock, K.E. McKay, R. Fong, \& N. Min (E.B., Psychology Dept. \& Neuropsychology Program, Queens College, CUNY, 65-30 Kissena Blvd., Flushing, NY 11367)
"Latent inhibition in preweanling rats" by P.J. Kraemer \& C.K. Randall (P.J.K., Dept. of Psychology, Univ. of Kentucky, Lexington, KY 40506)

"Effects of chronic treatment with 2,5-hexanedione on several behavioral measures in rats" by M.E. Pereira, C.I. Huang, \& I. Izquierdo (M.E.P., Setor de Bioquimica, CCNE-UFSM, Campus Universitario-Camobi, 97119 Santa Maria RS, Brasil)

" The hypoalgesia conditioned by a heat stressor with naloxone is nonopioid: Implications for the hypoalgesias conditioned by shock' by H. Foo (School of Psychology, Univ. of New South Wales, P.O. Box 1, Kensington 2033, Sydney, Australia) 